\title{
Plant non-coding RNAs and epigenetics
}

\author{
Jia-Wei Wang ${ }^{1,2^{*}}$ \& Yijun $\mathrm{Qi}^{3,4^{*}}$ \\ ${ }^{1}$ National Key Laboratory of Plant Molecular Genetics (NKLPMG), CAS Center for Excellence in Molecular Plant Sciences, Institute of Plant \\ Physiology and Ecology (SIPPE), Shanghai Institutes for Biological Sciences (SIBS), Shanghai 200032, China; \\ ${ }^{2}$ ShanghaiTech University, Shanghai 200031, China; \\ ${ }^{3}$ Center for Plant Biology, School of Life Sciences, Tsinghua University, Beijing 100084, China; \\ ${ }^{4}$ Tsinghua-Peking Center for Life Sciences, Beijing 100084, China
}

Received December 6, 2017; published online January 24, 2018

Citation: Wang, J.W., and Qi, Y. (2018). Plant non-coding RNAs and epigenetics. Sci China Life Sci 61, 135-137. https://doi.org/10.1007/s11427-017-9244-0

Epigenetics plays a large role in various aspects of plant biology, including development, response to biotic and abiotic stresses, silencing of transposable elements, and maintenance of genome stability. In plants, epigenetic regulation involves histone and DNA modifications and noncoding RNAs including small RNAs (sRNAs) and long noncoding RNAs (lncRNAs). This issue of Science China Life Sciences includes five review articles and eight research articles that explore functions and mechanisms of different types of sRNAs and lncRNAs, histone modification, and DNA methylation.

\section{NON-CODING RNAS: THE LONG AND THE SHORT OF THEM}

MicroRNAs (miRNAs) are a class of endogenous sRNAs that are processed from hairpin-structured precursors. miRNA expression often exhibits spatial and temporal specificity. $\mathrm{Xu}$ et al. present an expression atlas of Arabidopsis miRNAs by sequencing sRNA libraries generated from 27 different organ/tissue types (Xu et al., 2018). They found that different miRNA members within the same family have distinct spatial and temporal expression patterns and some miRNAs are produced from different arms of their hairpin

*Corresponding authors (Jia-Wei Wang, email: jwwang@sibs.ac.cn; Yijun Qi, email: qiyijun@tsinghua.edu.cn) precursors at different developmental stages. This work provides a rich resource for future investigation of miRNA functions in Arabidopsis. A subset of miRNAs has been identified to target the transcripts encoding plant resistance (R) proteins, the immune receptors that recognize pathogen effectors and trigger rapid defense responses. Li summarizes the recent progress in our understanding of how plant miRNAs reduce $R$ gene levels under normal conditions and allow induction of $R$ gene expression under stress conditions (Deng Y. et al., 2018).

Phased siRNAs (phasiRNAs) are generated from long RNA precursors (transcripts from protein-coding or noncoding genes) targeted by miRNAs at intervals of 21 or $24 \mathrm{nt}$ in plants. Interestingly, grass phasiRNAs are specifically expressed in reproductive organs. Yu et al. summarize current knowledge on phasiRNAs in male germ cells and their possible biological functions and mechanisms in anther development and male fertility (Yu et al., 2018). Transfer RNAderived small RNAs (tsRNAs) are generated from precursor or mature tRNAs. Zhu et al. review the recent progress in the discovery, biogenesis, and function of tsRNAs in higher plants (Zhu et al., 2018).

In addition to sRNAs, IncRNAs play critical roles in plant development and environmental acclimation. Deng et al. systematically analyze lncRNAs in five monocot and five dicot species (Deng P. et al., 2018). They found that the majority of lncRNAs have high sequence conservation at the intra-species and sub-species levels, similar to mRNAs. In- 
triguingly, they demonstrate that although highly diverged at the nucleotide level, some lncRNAs are conserved by position in the genome at the inter-species level and they are often co-expressed with their adjacent protein-coding genes. Wang et al. show that a lncRNA, named HIDl (hidden treasure 1), acts as a negative regulator of cotyledon greening in addition to its role in photomorphogenesis (Wang et al., 2018). HID1 acts downstream of phytochrome-interacting factors 3 (PIF3) during the dark-to-light transition. Genetic ablation of HID1 resulted in the elevated transcript levels of the protochlorophyllide oxidoreductases known to catalyze Pchlide to chlorophyllide conversion, and subsequently leading to an increased greening rate of etiolated seedlings. Lariat-derived circular RNAs (laciRNAs) are produced during pre-mRNA splicing. They are previously thought as by-products of mRNA processing in higher eukaryotes. Cheng et al. identify an Arabidopsis laciRNA that plays an essential role in gene regulation and plant development, suggesting that the production of laciRNAs may have biological significance (Cheng J. et al., 2018).

RNA editing is a type of post-transcriptional modification that includes nucleotide insertion/deletion or conversion. In higher plants, RNA editing usually occurs in plastids and mitochondria and plays important roles in organelle biogenesis, environmental adaptation and development. Yan et al. discuss the divergent roles of the plant RNA editing factors such as pentatricopeptide repeat (PPR) proteins, multiple organelle RNA editing factors (MORF, also known as RIP), organelle RNA recognition motif (ORRM) containing proteins, protoporphyrinogen IX oxidase 1 (PPO1) and organelle zinc finger 1 (OZ1)(Yan et al., 2018).

\section{HISTONE AND DNA MODIFICATIONS: AN EVER-GROWING PUZZLE}

In eukaryotes, epigenetic inheritance is mainly established by histone modification and DNA methylation. The covalent modifications on histone can be "read" by specific protein domains and subsequently trigger downstream signaling events. Liu et al. review recent structural studies on the recognition of the epigenetic marks on histones by reader proteins, and summarize the general and unique features of histone mark readers in plants (Liu et al., 2018).

EARLY FLOWERING IN SHORT DAYS (EFS, also known as SDG8) is the major contributor for H3K36 methylation in Arabidopsis (Kim et al., 2005; Zhao et al., 2005). Cheng et al. uncover a novel function of $E F S / S D G 8$ in embryogenesis (Cheng L. et al., 2018). They found that the mutation in $E F S / S D G 8$ causes large embryo and altered histone modification at an imprinted gene called MOP9.5. Lin et al. show that overexpression of the mutated forms of histone $\mathrm{H} 3(\mathrm{H} 3.1 \mathrm{~K} 36 \mathrm{M}$ and $\mathrm{H} 3.3 \mathrm{~K} 36 \mathrm{M})$ causes serious developmental defects (Lin et al., 2018). The H3K36M mutation inhibits the activity of $E F S /$ SDG8 and changes the modifications of endogenous histone $\mathrm{H} 3$, including acetylation at lysine $9(\mathrm{H} 3 \mathrm{~K} 9 \mathrm{ac})$, trimethylation at lysine 27 (H3K27me3), di- and tri-methylation at lysine 36 (H3K36me2 and H3K36me3).

The SU(VAR)-3-9-related protein family member SUVR2 is involved in transcriptional gene silencing in both RNAdependent and -independent pathways (Thorstensen et al., 2006). Luo et al. demonstrate that SUVR2 is mono-sumoylated in planta (Luo et al., 2018). The sumoylation of SUVR2 is required for the interaction of SUVR2 with the chromatin-remodeling proteins CHR19, CHR27, and CHR28 and transcriptional gene silencing.

In addition to histone modification, DNA methylation plays a vital role in gene expression and plant development. Chen et al. performed genome-wide profiling of DNA methylation at single-base resolution by whole-genome bisulfite sequencing in tomatoes (Chen et al., 2018). Taking the advantage of the tomato epimutant colourless non-ripening $(\mathrm{Cnr})$ and virus-induced gene silencing (VIGS) technology, they found that two genes, named SIDETI and SIPDS, are involved in the pigmentation in $\mathrm{Cnr}$ fruits.

These review and research articles underscore the importance of epigenetic mechanisms that regulate a variety of biological processes in plants. Our understanding of the functions and mechanisms of chromatin modifications and noncoding RNAs is still very limited. Further efforts and resources are needed to solve these ever-growing puzzles.

Compliance and ethics The author(s) declare that they have no conflict of interest.

Chen, W., Yu, Z., Kong, J., Wang, H., Li, Y., Zhao, M., Wang, X., Zheng, Q., Shi, N., Zhang, P., Zhong, S., Hunter, P., Tör, M., and Hong, Y. (2018). Comparative WGBS identifies genes that influence non-ripe phenotype in tomato epimutant Colourless non-ripening. Sci China Life Sci 61, 244-252.

Cheng, L., Shafiq, S., Xu, W., and Sun, Q. (2018). EARLY FLOWERING IN SHORT DAYS (EFS) regulates the seed size in Arabidopsis. Sci China Life Sci 61, 214-224.

Cheng, J., Zhang, Y., Li, Z., Wang, T., Zhang, X., and Zheng, B. (2018). A lariat-derived circular RNA is required for plant development in Arabidopsis. Sci China Life Sci 61, 204-213.

Deng, P., Liu, S., Nie, X., Weining, S., and Wu, L. (2018). Conservation analysis of long non-coding RNAs in plants. Sci China Life Sci 61, 190-198.

Deng, Y., Liu, M., Li, X., and Li, F. (2018). microRNA-mediated $R$ gene regulation: molecular scabbards for double-edged swords. Sci China Life Sci 61, 138-147.

Kim, S.Y., He, Y., Jacob, Y., Noh, Y.S., Michaels, S., and Amasino, R. (2005). Establishment of the vernalization-responsive, winter-annual habit in Arabidopsis requires a putative histone H3 methyl transferase. Plant Cell 17, 3301-3310.

Lin, G., Zhou, Y., Li, M., and Fang, Y. (2018). Histone 3 lysine 36 to methionine mutations stably interact with and sequester SDG8 in $\mathrm{Ar}$ abidopsis thaliana. Sci China Life Sci 61, 225-234.

Liu, R., Li, X., Chen, W., and Du, J. (2018). Structure and mechanism of plant histone mark readers. Sci China Life Sci 61, 170-177. 
Luo, Y.X., Han, Y.F., Zhao, Q.Y., Du, J.L., Dou, K., Li, L., Chen, S., and He, X.J. (2018). Sumoylation of SUVR2 contributes to its role in transcriptional gene silencing. Sci China Life Sci 61, 235-243.

Thorstensen, T., Fischer, A., Sandvik, S.V., Johnsen, S.S., Grini, P.E., Reuter, G., and Aalen, R.B. (2006). The Arabidopsis SUVR4 protein is a nucleolar histone methyltransferase with preference for monomethylated H3K9. Nucleic Acids Res 34, 5461-5470.

Wang, Y., Li, J., Deng, X. W., and Zhu, D. (2018). Arabidopsis noncoding RNA modulates seedling greening during deetiolation. Sci China Life Sci 61, 199-203.

Xu, L., Hu, Y., Cao, Y., Li, J., Ma, L., Li, Y., and Qi, Y. (2018). An expression atlas of miRNAs in Arabidopsis thaliana. Sci China Life Sci $61,178-189$.

Yan, J., Zhang, Q., and Yin, P. (2018). RNA editing machinery in plant organelles. Sci China Life Sci 61, 162-169.

Yu, Y., Zhou, Y., Zhang, Y., and Chen, Y. (2018). Grass phasiRNAs and male fertility. Sci China Life Sci 61, 148-154.

Zhao, Z., Yu, Y., Meyer, D., Wu, C., and Shen, W.H. (2005). Prevention of early flowering by expression of FLOWERING LOCUS $\mathrm{C}$ requires methylation of histone H3 K36. Nat Cell Biol 7, 1256-1260.

Zhu, L., Ow, D.W., and Dong, Z. (2018). Transfer RNA-derived small RNAs in plants. Sci China Life Sci 61, 155-161.
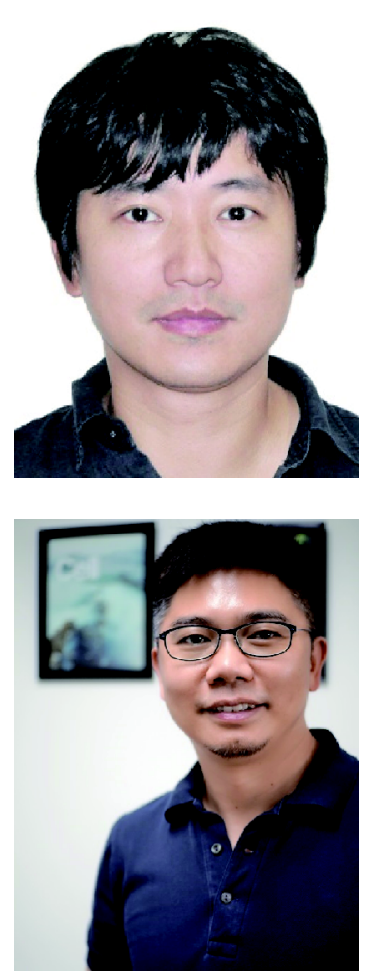

\section{Biographical Sketch}

Dr. Jia-Wei Wang was born in Shanghai and graduated from the Department of Life Science and Technology, Shanghai Jiao Tong University in 1999. He received a Ph.D. degree at Shanghai Institutes for Biological Sciences, Chinese Academy of Sciences (CAS) in 2005. He went to Germany as a postdoc in 2005 and received postdoctoral training at Max Planck Institute for Developmental Biology. His independent research began from 2011 and he is currently a principal investigator at National Key Laboratory of Plant Molecular Genetics (NKLPMG), CAS Center for Excellence in Molecular Plant Sciences, Institute of Plant Physiology and Ecology (SIPPE), CAS. The main research focus of his group is plant life cycle and developmental timing.

Dr. Yijun Qi is a Professor in School of Life Sciences and Center for Plant Biology at Tsinghua University in Beijing. He completed a Ph.D. in plant virology at Zhejiang University. He is an expert on RNA silencing in plants. Work in his laboratory includes investigating the functions of small RNAs and long non-coding RNAs, biochemical and molecular analysis of Argonaute complexes, and RNA-directed repair of DNA double-strand breaks. 